\title{
Unternehmen droht Fehlstart bei SEPA
}

$\mathrm{Ab}$ dem 1. Februar 2014 müssen Überweisungen und Lastschriften durchgängig im neuen SEPA-Format abgewickelt werden. Transaktionen im alten Format dürfen ab diesem Zeitpunkt nicht mehr ausgeführt werden. Doch gut jedes fünfte Unternehmen hat bislang nur vage Vorstellungen von der Umstellung des Zahlungsverkehrs auf SEPA (Single Euro Payments Area). Rund 30 \% planen erst zum Stichtag am 1. Februar 2014 die ausschließliche Nutzung der neuen Überweisungsform. Das geht aus der „Bestandsaufnahme zur SEPA-Migration in Deutschland" des Forschungsinstituts ibi research an der Universität Regensburg in Kooperation mit dem Bundesverband Informationswirtschaft, Telekommunikation und neue Medien e. V. (BITKOM) hervor. Befragt wurden deutsche Unternehmen, Behörden und Vereine. Von der Umstellung betroffen sind sämtliche Abteilungen, die mit bargeldlosem Zahlungsverkehr zu tun haben, wie etwa IT, Buchhaltung, Vertrieb, Einkauf oder die Personalabteilung.
„Die Folgen einer verspäteten Umstellung auf SEPA können existenziell sein, vielen unvorbereiteten Unternehmen droht ab Februar 2014 die Zahlungsunfähigkeit“, sagt BITKOM-Experte Steffen von Blumroeder. Jedes vierte Unternehmen (27 \%) gibt an, im Falle fehlender Lastschrifteingänge innerhalb von fünf Tagen Liquiditätsprobleme zu bekommen.

\section{Mehr zum Thema}

Müller, R. H.: SEPA, eine aktuelle Herausforderung für das Forderungsmanagement, in: Müller, R. H.: Erfolgreiches Forderungsmanagement - Effektive Lösungen unter Berücksichtigung der SEPA-Umstellung, Wiesbaden 2013, S. 233 - 254. sipip $^{*}$ www.springerprofessional.de/4686038

Bianca Baulig, Wiesbaden

\section{CFOs setzen für den Jahresabschluss verstärkt auf Zeitarbeitskräfte}

Geht das laufende Geschäftsjahr zu Ende, laufen die Vorbereitungen für die Erstellung des Jahresabschlusses auf Hochtouren. Häufig werden die Zeitressourcen im Rechnungswesen knapp. Überstunden sind in nahezu allen Finanzteams die Regel. Immer mehr Finanzvorstände erwägen daher den Einsatz von Zeitarbeitskräften, um die Arbeitsbelastung besser verteilen zu können.

Dies ergab eine Studie des Personaldienstleisters Robert Half, an der rund 200 CFOs teilgenommen haben. Das Ergebnis zeigt, dass bei fast der Hälfte der Befragten (43\%) die Finanzabteilungen während der Jahresabschlusserstellung mit knappen Zeitressourcen kämpfen. 27 \% der CFOs empfinden sinkende Motivation innerhalb der Abteilung und mangelndes Fachwissen als weitere Hürden. Jeder dritte Finanzvorstand (33 \%) hält die Zusammenarbeit mit Abschlussprüfern für aufwendig.

Um diese Hürden zu überwinden, setzen CFOs verstärkt auf Zeitarbeitskräfte. So ziehen laut Robert Half 60 \% der be- fragten CFOs die Möglichkeit in Betracht, interimsweise qualifizierte Fachkräfte für den Jahresabschluss zu engagieren. $14 \%$ planen sogar fest damit, für den Jahresabschluss 2013 Zeitpersonal einzustellen. Im Mittelstand ist diese Lösung besonders beliebt: Hier sprechen sich 68 \% der Unternehmen für den Einsatz qualifizierter Zeitarbeiter aus.

Mehr zum Thema

Gerner, G.: Zeitarbeit: Element einer Beschäftigungsstrategie zur Überwindung des Fachkräftemangels, in: Bornewasser, M./Zülich, G.: Arbeitszeit - Zeitarbeit - Flexibilisierung der Arbeit als Antwort auf die Globalisierung, Wiesbaden 2013, S. $77-97$.

SSTP $^{*}$ www.springerprofessional.de/3686480

Sylvia Meier, Wiesbaden 